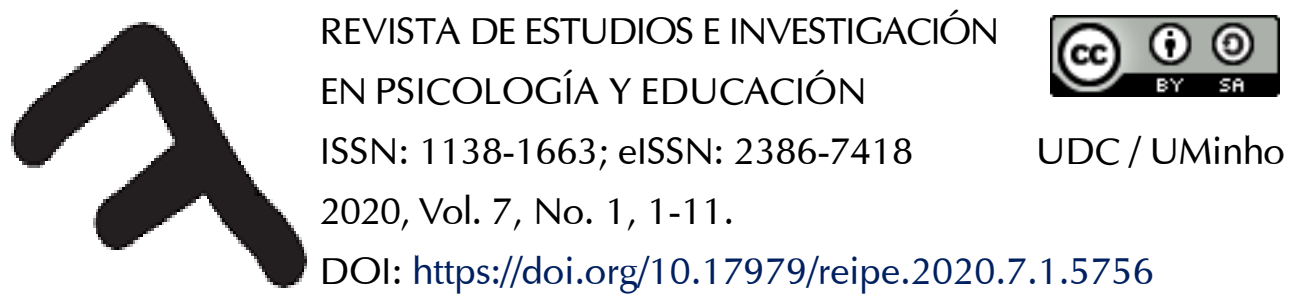

\title{
Percepción subjetiva y socialización de las relaciones románticas de pareja en futuros y futuras docentes
}

\author{
Subjective perception and socialisation of romantic relationships among student \\ teachers
}

\author{
Esther Rivas-Rivero (D), Enrique Bonilla-Algovia \\ Universidad de Alcalá
}

\begin{abstract}
Resumen
Las relaciones de pareja suelen ser una fuente de bienestar y un proyecto de vida compartida a partir de la relevancia que se otorga a esta forma de organización social, sin embargo, el ideario del amor romántico tiene consecuencias negativas que tienen su origen en los distintos agentes de socialización. El presente estudio cualitativo analiza las percepciones de 34 futuros y futuras docentes, cuya edad media es de 21.32 años $(\mathrm{DT}=3.121)$, respecto a las relaciones de pareja y el amor romántico. Los resultados muestran que el compromiso y la reciprocidad son indicadores importantes de bienestar propio y compartido, si bien, se encontraron respuestas asociadas al malestar relacionadas con los imperativos sociales del amor romántico. Además, las personas participantes aludieron a los celos y a conductas de control por parte de las parejas con bastante frecuencia, principalmente entre las mujeres. Visibilizar el significado que se da al amor romántico y a las relaciones de pareja podría favorecer el desarrollo de una perspectiva crítica respecto a cómo se han asimilado estos modelos relacionales entre quienes socializarán a las futuras generaciones.
\end{abstract}

Palabras clave: influencia de pares; comportamiento social; patrones socioculturales

\begin{abstract}
Relationships are usually perceived as a source of well-being and the basis for a shared life plan owing to their importance as a form of social organisation. However, the ideology of romantic love promoted by the different agents of socialisation can have negative consequences. The aim of this qualitative study is to analyse perceptions regarding relationships and romantic love among 34 student teachers with an average age of 21.32 years $(\mathrm{SD}=3.121)$. The results show that commitment and reciprocity are important indicators of individual and shared well-being, although some responses indicated discomfort in relation to the social imperatives of romantic love. Participants also made frequent reference to certain negative aspects of relationships, such as jealousy and controlling behaviours by mainly female partners. Greater awareness and recognition of the meaning and significance attributed to romantic love and relationships could help to examine critically how these relational models have been assimilated by those responsible for socialising future generations.
\end{abstract}

Keywords: partner influence; social behaviour; sociocultural patterns

Esther Rivas-Rivero (Dorcid.org/0000-0001-5092-1260 y Enrique Bonilla-Algovia Dorcid.org/0000-0003-1667-1880: Departamento de Ciencias de la Educación. Universidad de Alcalá. Calle San Cirilo, s/n. 28801 Alcalá de Henares, España.

Correspondencia relativa a este artículo: Esther Rivas - esther.rivas@uah.es, Enrique Bonilla - enrique.bonilla@uah.es 
Uno de los objetivos prioritarios promovidos por la UNESCO para la Agenda Educación 2030 es la implementación de medidas que contemplen la igualdad y la perspectiva de género en todos los ámbitos educativos y en la formación del profesorado (Arango y Corona-Vargas, 2016). En este sentido, en España se viene produciendo un cambio en la socialización de las nuevas generaciones (Subirats, 2017). No obstante, la teoría de la socialización diferencial considera que niños y niñas son diferentes, lo que trae como consecuencia el desarrollo de roles diferenciados en su vida adulta (Ferrer y Bosch, 2013). Desde las instituciones educativas, el profesorado tiene una importante labor como agente de socialización en la construcción del género y en el análisis crítico de los roles que se transmiten en diversos ámbitos que legitiman la desigualdad entre hombres y mujeres (Arango y Corona-Vargas, 2016). Además, las estadísticas respecto a la violencia contra las mujeres en España muestran cifras que hacen que la formación y la intervención social y educativa sean una necesidad (Carbajosa y Boira, 2013).

Algunos referentes legislativos están favoreciendo la igualdad de género y la coeducación (Carretero y Nolasco, 2019). La Ley Orgánica 1/2004, de 28 de diciembre, de Medidas de Protección Integral contra la Violencia de Género, establece que se promoverá la formación permanente del profesorado en materia de igualdad. La Ley Orgánica 2/2006, de 3 de mayo, de Educación (LOE), en su artículo 2 contempla que uno de los fines del Sistema Educativo es el respeto de los derechos y libertades fundamentales, así como la igualdad y la no discriminación entre hombres y mujeres. Recientemente, el Decreto 32/2019, de 9 de abril, por el que se regula la convivencia en los centros docentes de la Comunidad de Madrid, recoge la prevención de la violencia de género, la igualdad y la no discriminación, que habrá de reflejarse en los respectivos planes de convivencia a través de actuaciones específicas y medidas correctoras. Este avance en materia de género no ha estado exento de algún retroceso. Concretamente, para Subirats (2017), la Ley Orgánica 8/2013, de 9 de diciembre para la Mejora de la Calidad Educativa (LOMCE), es un reflejo de los aspectos más retrógrados de nuestra sociedad en materia de género, pues en su artículo 84.3 se legitima la educación separada por sexos.

En esta línea, desde la socialización diferencial tradicional, se espera de los hombres proyección en la esfera pública y se les ha educado en que la gratificación y la autoestima provengan de dicha proyección, fomentando su independencia y reprimiendo el ámbito afectivo. Por el contrario, las mujeres son educadas para el desempeño de roles pertenecientes al contexto privado, coartando sus ambiciones y desarrollando la dependencia y el afecto hacia otros (Ferrer y Bosch, 2013; Vázquez y Rivas, 2017). Además, dicha socialización diferencial afecta a las relaciones de pareja respecto a qué significa enamorarse, cuáles son los imperativos sociales sobre el amor romántico y cómo debe ser una relación de pareja (Ferrer, Bosch, Navarro, Ramis, y García, 2008).

La investigación respecto a las relaciones románticas en la adolescencia y en la juventud es relativamente reciente, aunque en las teorías del desarrollo está ampliamente aceptado datar el inicio de las relaciones románticas en torno a los 13 años (González y Molina, 2018). En esta línea, el trabajo de Pazos, Oliva y Hernando (2014), realizado con jóvenes andaluces de edades comprendidas entre los 14 y los 20 años, muestra que el $51 \%$ había mantenido, al menos, una relación de pareja, teniendo lugar la primera relación a una edad media de 13 años. No obstante, existe disparidad respecto a situar el inicio de las relaciones románticas a una edad concreta, ya que la variabilidad podría proceder de diversos factores como la escasez de apoyo social en el grupo de pares, el bajo rendimiento académico o problemas en el seno familia (Boisvert y Poulin, 2016). Además, un inicio precoz de las relaciones románticas podría derivar en desventajas y desajustes emocionales por no estar preparados para las demandas relacionales (Bisquert-Bover, Giménez-García, Gil-Juliá, MartínezGómez, y Gil-Llario, 2019; González y Molina, 2018; Valle y De la Villa, 2018).

Hay que añadir que los adultos jóvenes describen las relaciones que mantuvieron en la adolescencia como más conflictivas y problemáticas que las relaciones del presente (Shulman y Kipnis, 2001). Con la edad, los miembros de la pareja parecen estar menos interesados en la obtención del beneficio propio y se centran en aumentar las ganancias mutuas (Lantagne y Furman, 2017). No obstante, las conductas y modelos en las relaciones de pareja proceden de la influencia del entorno, y tienen su base en las teorías del aprendizaje social (Wheeler, Killoren, Whiteman, Updegraff, y McHale, 2016). Además, a medida que las relaciones aumentan 
en duración, podrían emerger distintos rasgos, como el apoyo mutuo o los conflictos en las interacciones, el control de la pareja y los celos (Lantagne y Furman, 2017).

Por otra parte, que las relaciones de pareja se sobrevaloren podría deberse a la influencia de los mitos del amor romántico (Bonilla-Algovia y Rivas-Rivero, 2018). Estos proceden del imaginario social acerca de la idealización del amor y representan una serie de creencias socialmente compartidas con implicaciones diferenciadas para hombres y mujeres (Bonilla y Rivas, 2018). La idealización de la relación romántica provoca que el concepto "tú/yo" pase a denominarse "nosotros", lo que puede desarrollar dependencia emocional bajo la necesidad de encontrar esa prescripción social dentro de la pareja (Kawamichi et al., 2016; Valle y De la Villa, 2018). Además, los imperativos sociales en las relaciones de pareja heterosexuales podrían impedir a las mujeres ser ellas mismas, ya que perderían la propia identidad con el objetivo de obtener el afecto del hombre (Rodríguez-Castro, Lameiras-Fernández, y Carrera, 2015).

Algunas investigaciones han demostrado la existencia de diferencias respecto a qué se entiende por amor entre hombres y mujeres, de modo que ellas manifiestan una mayor predisposición al sacrificio, mientras que los hombres, aunque conceden importancia a disponer de una pareja (Ferrer, Bosch, y Navarro, 2010), priorizan su proyecto vital y personal (Bosch, Herrezuelo, y Ferrer, 2019). Además, las mismas autoras señalan que existe una mayor idealización de las relaciones románticas en las mujeres que en los hombres, ya que estos se identifican en menor medida con la entrega total o entienden que dicha entrega se dirija hacia su propio beneficio (Bosch et al., 2019). También se ha encontrado que, en el marco de los imperativos sociales respecto a las relaciones románticas, emergen conductas relacionadas con la manipulación y el control en el seno de la pareja (Brewer y Abell, 2015; Ináncsi, Lang, y Bereczkei, 2015). En opinión de García-Carpintero, RodríguezSantero y Porcel-Gálvez (2018), estos patrones comportamentales, derivados del imaginario sobre el amor, reafirmarían en los hombres su masculinidad; en las mujeres se orientaría a afrontar estados de inseguridad, produciéndose mayores niveles de dependencia y malestar en ellas. Además, se ha señalado que el control y la manipulación son formas de ejercer la violencia (Carton y Egan, 2017). Es por esto por lo que las mujeres no identifican la violencia en el noviazgo pese a que las conductas de control y manipulación sean fuentes de malestar, ya que dicho control podría entenderse como una demostración de amor. Asimismo, aunque la desconfianza y los celos son fuentes de conflicto en una relación romántica (Brewer y Abell, 2017), bajo la idealización del amor, pueden interpretarse como una demostración de afecto, aun tratándose de una conducta posesiva y de coerción (Bonilla-Algovia y Rivas-Rivero, 2018).

Por todo ello, la educación socioafectiva y el concepto del amor romántico están siendo objeto de estudio y están suscitando interés entre la comunidad científica en la prevención de la violencia de pareja (Pascual, 2016), ya que la idealización del amor podría favorecer la tolerancia hacia el maltrato bajo el marco de "el amor todo lo puede" (mito de la omnipotencia) o que es normal "sufrir por amor" (Ferrer y Bosch, 2013). La socialización diferencial y el aprendizaje respecto a qué se entiende por amor favorecen la aceptación por parte de las nuevas generaciones de muchos mitos y falacias sobre el amor romántico que, unido a la no identificación sobre qué se considera maltrato, hacen que sea relevante abordar estos aspectos (De Miguel, 2015). Que hombres y mujeres sean socializados bajo concepciones diferenciadas del amor puede estar legitimando la desigualdad de género en las relaciones de pareja (Bonilla y Rivas, 2018a), y podría tener graves consecuencias para el bienestar de las mujeres (Rivas y Bonilla, 2020; Rivas, Bonilla, y Vázquez, 2020). Por ello, las instituciones educativas tienen un papel muy relevante en la deconstrucción del amor romántico y en el desarrollo de una socialización basada en la igualdad de género. En este sentido, el profesorado es clave y sobre este recae la responsabilidad de modificar los modelos tradicionales basados en la desigualdad entre mujeres y hombres (Bonilla-Algovia y Rivas-Rivero, 2019).

El objeto de este estudio es analizar las percepciones sobre las relaciones de pareja y el amor romántico en una muestra de futuros y futuras docentes desde un enfoque cualitativo, ya que permite la identificación y visibilización de tales construcciones, así como identificar en dichas percepciones la influencia de la socialización diferencial. 


\section{Método}

El presente estudio se enmarca dentro de la metodología cualitativa, con la que se busca la interpretación de los significados relativos a un contexto para comprender la perspectiva de las personas participantes partiendo de sus historias personales en el marco de la vida cotidiana en circunstancias concretas (Carrasco y Calderero, 2007; Forni, 2010). El enfoque cualitativo se basa en la comprensión de la conducta humana, entendida como acción social, en el que cobra especial importancia la categorización para contextualizar los hechos a través del análisis del discurso de las personas involucradas como eje del conocimiento social (Carrasco y Calderero, 2007; Fassio, 2018). Para ello hace uso de técnicas como la semiótica, el análisis del relato y el análisis fonológico respecto al fenómeno objeto de estudio (Denzil y Lincoln, 2011). Por lo tanto, la metodología cualitativa permite el acercamiento en profundidad y la visibilización de nuevas temáticas que complementan el conocimiento proporcionado por otras fuentes teóricas respecto a las creencias sobre las relaciones de pareja y los mitos del amor romántico (Bonilla y Rivas, 2018a; Bosch et al., 2019; Ferrer y Bosch, 2013; Papp, Liss, Erchull, Godfrey, y Waaland-Kreutzer, 2017; Pascual, 2016; Rodríguez-Castro et al., 2015).

\section{Participantes}

En la investigación participaron 34 jóvenes de la Comunidad de Madrid, 9 hombres y 25 mujeres, que se encontraban cursando titulaciones de grado en Educación Primaria en la Universidad de Alcalá. El tipo de muestreo fue de casos homogéneos, el cual busca la descripción de un subgrupo en profundidad para la confrontación de perspectivas comunes (Patton, 1987), en este caso, su experiencia en las relaciones románticas de pareja. Los criterios de inclusión para formar parte del estudio fueron: 1) ser mayor de edad, 2) tener o haber tenido al menos una relación de pareja y 3) estar matriculado en estudios de Magisterio.

La edad media de la muestra era de 21 años, siendo superior en los hombres que en las mujeres. La mayoría se adscribía a la clase social media. Aunque la totalidad de la muestra había tenido pareja anteriormente, menos de la mitad mantenía una relación en el momento del estudio. Cabe señalar que quienes participaron en los grupos focales cursaban el Grado de Magisterio en Educación Primaria.

\section{Instrumentos}

Concretamente, los grupos focales consisten en el establecimiento de grupos para discutir uno o varios temas propuestos por la persona que modera las interacciones, de modo que los distintos integrantes puedan colaborar para obtener información a través de la confrontación de ideas, así como obtener su perspectiva sobre las actitudes, experiencias o creencias (De Lara y Ballesteros, 2007; Gil, 2011). En total se formaron cuatro grupos focales homogéneos en cuanto al sexo: Grupo 1 (9 hombres); Grupo 2 (9 mujeres), Grupo 3 (8 mujeres) y Grupo 4 ( 8 mujeres). La distribución de las personas participantes fue intencional, y se optó por la creación de grupos homogéneos con el fin de controlar que las respuestas no estuviesen sesgadas por la presión o deseabilidad social ante la presencia de miembros del sexo opuesto. Cabe señalar que la baja presencia de hombres respecto a mujeres se debe a la baja matriculación de varones en las titulaciones de Educación y la feminización de estos estudios.

Se utilizó un guion de preguntas que permitió abordar los objetivos de la investigación en las que se encontraban cuestiones como las expectativas respecto a las relaciones de pareja y el significado que otorgaban al amor. Las entrevistas tuvieron una duración que osciló entre 70 y 120 minutos, que varió en función de la saturación de la información en cada grupo (grounded theory).

\section{Procedimiento}

El profesorado de la Universidad de Alcalá informó al alumnado respecto a la investigación que se iba a realizar. A partir de las personas que voluntariamente se adscribieron al estudio, se seleccionaron las que cumplían con los criterios de inclusión muestral. Se informó a las personas participantes de los objetivos y las características de la investigación, y se les facilitó un cuestionario en el que se recogía el consentimiento 
informado y los datos sociodemográficos. Con todo, se diseñaron los grupos focales. Las sesiones se llevaron a cabo en las instalaciones de la Universidad de Alcalá y se grabaron mediante dispositivos de recogida de audio. Los temas se codificaron para su posterior procesamiento y se creó un sistema de categorías. Esto permitió un acceso rápido a las mismas que facilitó el análisis del contenido. Los datos se organizaron en nodos de información en función de sus diferencias y similitudes. Se comparó la información codificada y se seleccionaron las citas que mejor representaban las categorías de análisis, que han sido transcritas de forma literal.

Los puntos suspensivos en la transcripción indican una pausa o indecisión en la última palabra; el paréntesis con puntos suspensivos (...) indica la eliminación de parte de la cita original. Los datos se procesaron y analizaron de forma anónima, asignando un código a cada cita que representa el grupo focal de referencia (G1, G2, G3 o G4), el sexo (H-M) y el número asignado a cada participante $(1,2,3 \ldots)$.

\section{Análisis de datos}

Se creó un equipo de personas expertas de la universidad con experiencia previa en investigación cualitativa. Mediante un procedimiento deductivo, el primer paso fue transcribir todas las intervenciones de los grupos focales e identificar temas potenciales. A partir de esto se estableció un listado unificado de temas a codificar. En el segundo paso se generaron diversas categorías y se analizaron discrepancias. Finalmente, se triangularon y unificaron las categorías y se seleccionaron los fragmentos más representativos.

En la Tabla 1 se muestran los significados y sistemas de categorías que las personas que participaron del estudio otorgaban al amor romántico y a las relaciones de pareja. Se extrajeron un total de nueve temas agrupados en torno a dos bloques referidos al "bienestar" y el "malestar" en dichas relaciones. Por un lado, en cuanto al bloque de bienestar, las personas participantes concebían como rasgo positivo la idea o mito relacionado con que "el amor todo lo puede" (mito de la omnipotencia) y la idea relacionada con que "no es necesario tener pareja para ser feliz". En cuanto al bloque relacionado con el "malestar", de este se desprendieron más subcategorías. En primer lugar, se encuentra la "idealización del amor romántico" del que derivaron creencias asociadas con la "necesidad de tener pareja" (mito del emparejamiento), relacionada con la "dependencia emocional", así como con el mito "donde hay amor, hay sufrimiento". Otra subcategoría perteneciente al bloque del malestar en la pareja es la que se basaba en respuestas relativas a las altas expectativas respecto a la "reciprocidad", entendida como una exigencia en las relaciones de pareja (mito de la entrega total) y de la que se extrajeron respuestas vinculadas a los sentimientos de "culpa". Asimismo, dentro del bloque de malestar también se refirieron a "los celos", percibidos por buena parte de la muestra como un aspecto negativo y no como una "demostración de amor". Finalmente, hicieron alusión a situaciones y experiencias en las que se había ejercido "maltrato" hacia las mujeres, siendo este el último segmento analizado.

\section{Tabla 1}

Sistema de categorías y subcategorías para analizar las percepciones sobre las relaciones de pareja y el amor romántico

\begin{tabular}{lcc}
\hline \multicolumn{1}{c}{ Categorías } & \multicolumn{1}{c}{ Subcategorías } & Segmentos \\
\hline $\begin{array}{l}\text { 1. Bienestar en las relaciones } \\
\text { románticas de pareja }\end{array}$ & $\begin{array}{l}\text { 1.1. El amor todo lo puede (mito de la } \\
\text { omnipotencia) } \\
\text { 1.2. Se puede ser feliz sin tener pareja }\end{array}$ & \\
\hline & $\begin{array}{l}\text { 2.1. Idealización del amor romántico } \\
\text { Mareja }\end{array}$ & $\begin{array}{l}\text { 2.1.1. Dependencia. } \\
\text { 2.1. Donde hay amor, hay } \\
\text { sufrimiento }\end{array}$ \\
$\begin{array}{l}\text { 2.2. Reciprocidad como exigencia (mito } \\
\text { de la entrega total) }\end{array}$ & $\begin{array}{l}\text { 2.2.1. Culpa } \\
\text { 2.3. Celos } \\
\text { 2.4. Maltrato (falacia de la vinculación } \\
\text { amor/maltrato) }\end{array}$ & \\
\hline
\end{tabular}




\section{Resultados}

A continuación, se exponen las categorías señaladas y los fragmentos más representativos respecto a las percepciones de las personas participantes en torno a los mitos del amor romántico y las relaciones de pareja.

\section{El amor todo lo puede (mito de la omnipotencia)}

La asociación entre tener pareja y el desarrollo de sentimientos de felicidad es una opinión muy extendida entre las personas participantes, de modo que, además del desarrollo de bienestar personal procedentes del compromiso en la relación, también se concibe que el amor y las relaciones de pareja son un recurso para superar problemas. En este sentido, las respuestas de las personas participantes apuntan al mito de "el amor todo lo puede" como algo subjetivamente positivo: "El amor es algo grande y con lo que se puede luchar para que todo salga bien" (G4, M 2). El fragmento que se expone a continuación hace aún más explícito dicho mito:

Fuera de los cánones que hay en la sociedad, sobre todo en la occidental, que es en la que estamos, [lo que espero de una relación es] felicidad, pasarlo bien, resolver problemas, porque los problemas surgen, pero con el amor $\mathrm{t}$ tal, yo creo que se pueden resolver. Es una buena herramienta $(\mathrm{G} 1, \mathrm{H} 7)$.

\section{Se puede ser feliz sin tener pareja}

También se hallaron percepciones asociadas con el bienestar basándose en la autonomía y en restar relevancia a tener una relación de pareja. En este grupo están las respuestas de quienes rechazaron el mito del emparejamiento, siendo la autonomía y la independencia valores que tradicionalmente se han transmitido al género masculino bajo la socialización diferencial. Cabe señalar que las participantes que proporcionaron respuestas que aludían a la no necesidad de mantener una relación romántica no tenían pareja en el momento de la investigación, por lo que podrían verse en menor medida condicionadas por el ideario social respecto a tener dichas relaciones de pareja: "Yo, mi felicidad la pongo en mí. (...). Nadie tiene mi felicidad (...). Depende de mí y sola estoy muy bien" (G2, M 7). "Puedes estar bien sola (...). No es una necesidad, sólo un complemento" (G4, M 1).

Cabe señalar que en uno de los casos se identifica una crítica a la presión social que recibe del entorno en cuanto a tener una relación de pareja:

Hay más gente que piensa que me ve más incompleta a mí por no tener pareja que yo misma. Se da mucha importancia a tener pareja y a lo mejor quieres estar sola o estás en un momento en el que te sientes bien contigo misma, aunque tienes que sentirte igualmente bien contigo misma teniendo pareja (G4, M4).

\section{Idealización del amor romántico}

Desde un punto de vista opuesto, y como fuente de malestar, se dieron opiniones basadas en la "idealización el amor romántico". En el ejemplo que se muestra a continuación, de la ausencia de amor o la "necesidad de tener pareja" podría emerger un bloqueo en el desarrollo personal, ya que se sentiría incompleta. Además, se aprecia el rechazo o resistencia a proyectar un futuro y una vida en solitario: "Yo me sentiría incompleta (...). ¿Toda una vida sin pareja? (G2, M 4).

Se identificó en algunas participantes percepciones respecto a que las relaciones románticas son un mecanismo con el que aumentar la autoestima. Desde esta perspectiva, podría desarrollar sentimientos de "dependencia emocional" ante la falta de reconocimiento propio y la necesidad de ser valorada por la pareja: "Si yo me encuentro mal conmigo misma, me encuentro fea y tengo pensamientos súper tóxicos, en ese momento me gustaría tener a alguien a mi lado que me dijera que valgo, que me quiere" (G4, M1).

Algunas de las personas participantes entienden que del ideario del amor también se desprenden sentimientos de malestar que influyen en las personas. Una de las participantes afirma que uno de los aspectos negativos que se circunscribe al amor es la idealización de este, de lo que se desprende la categoría referida al mito del amor romántico "donde hay amor, hay sufrimiento": 
El amor es pasarlo mal. Yo lo que espero de una relación amorosa es felicidad y durabilidad. Pero la experiencia me ha enseñado que también lo que tengo que esperar es dolor y sufrimiento porque, en un porcentaje muy amplio, las relaciones de pareja se acaban (G3, M5).

\section{Reciprocidad como exigencia (mito de la entrega total)}

Asimismo, uno de los participantes reporta que en las relaciones de pareja ha de haber altos niveles de "reciprocidad". Además, se aprecia cierta exigencia respecto a esa concepción del amor al considerarlo "vital" o que dicha reciprocidad tenga que ser lo "mínimo" que se ha de esperar de una relación. Desde estos imperativos, y unas expectativas elevadas que implican altas demandas hacia la otra persona, podrían surgir sentimientos de frustración o decepción:

Me parece vital que en una relación te comportes como te gustaría que se comportasen contigo.

Es decir, si tú quieres estar con una persona que sea sincera, que te escuche, que esté cuando tengas

problemas, lo mínimo que tienes que hacer es hacer exactamente lo mismo (G1, H8).

Además de la reciprocidad, las relaciones pueden ser una forma de control que merman el desarrollo y la conducta de la persona como sujeto individual. Así, algunas participantes reconocieron que podría ser una herramienta entre quienes ejercen manipulación, que derivaría en sentimientos de "culpa" cuando se busca autonomía. Cabe señalar que el desarrollo de sentimientos de culpa ha sido referido por mujeres. Hay que añadir que, además de la auto-punición percibida, se aprecia subordinación, ya que se desprende la necesidad de obtener el consentimiento de la otra persona, que se correspondería con las formas de socializar a las mujeres respecto a "ser para otros":

Hay personas que utilizan su malestar para controlarte, de modo que acabas diciendo: es que mi pareja está mal, cómo voy a salir de fiesta. A la semana, es que mi pareja ha discutido con su padre, cómo voy a quedar con mis amigas. Y así, semana tras semana ... te hace sentir culpable (G4, M5).

El hecho de enfadarse y de hacerte sentir a ti mal, ya es como... (...). A no ser que, si él coge y está bien y te dice, pues mira sí, vete y pásatelo bien, y te apoya, tú vas a estar bien, porque estás bien con tus amigas y bien con tu pareja (G3, M3).

\section{Los celos no son una demostración de amor}

Por otro lado, en las relaciones, bajo la exigencia de ser estables, se pueden desarrollar sentimientos de posesión y un sentido de pertenencia de la otra persona. Bajo el marco de los mitos del amor romántico, los celos son entendidos como una demostración de amor, sin embargo, para los hombres, los celos son interpretados como una emoción negativa que tiene que ser bien gestionada. Es importante reseñar la normalización de estos cuando se alude a la falta de control.

El celo, lo que es el celo, es un sentimiento, es una emoción. Y una emoción no se puede controlar. Y ahí viene el problema. Si tú tienes la suficiente inteligencia emocional, esa emoción sabes llevarla... o simplemente hablarlo con ella. Pero el ser celoso o sentir alguna vez celos es algo totalmente... Es que es una emoción, es imposible no tenerla (G1, H6).

Algunas de las personas entrevistadas consideran que es la falta de autocontrol la que hace que los celos influyan negativamente en la relación. En la siguiente afirmación se aprecia un sentido crítico de los celos como construcción social en el ideario del amor y, aunque reconoce haberlos experimentado en el pasado, indica no tenerlos en la relación de pareja que mantiene en el presente: "Yo creo que los celos es algo que nos han enseñado. Una construcción social, realmente. Porque yo antes era celosa y he conseguido quitármelos. Y realmente no tengo ningún tipo de celos de mi pareja (...). Además, me parecen muy tóxicos" (G2, M5).

\section{Maltrato en las relaciones de pareja}

No se han encontrado respuestas que vinculen el "amor y el maltrato", que es un mito referido en la literatura científica; sin embargo, han señalado manifestaciones de violencia en relaciones del pasado o han identificado conductas en las que está presente el maltrato en relaciones de personas del entorno. 
Yo he roto parejas por malos tratos, porque no me han tratado bien. Por ejemplo, mi pareja de los 15 años, cuando hacía algo que a él no le parecía bien, me gritaba mucho (...); luego me pedía perdón y me decía que le había puesto nervioso. Y llegó un punto en el que las discusiones llegaban a ser tan horribles que no quería escucharle (G3, M5).

Me molesta mucho haber oído hablar de todo lo que rodea al feminismo tarde, porque he tenido amigas en relaciones muy tóxicas y cuando me hablaban de sus relaciones, sabía que sus parejas las hacían mal. Sin embargo, no he sabido hasta qué punto ha sido tan fuerte ese maltrato y me fastidia no haber tenido las herramientas para haberlas ayudado en ese entonces" (G4, M4).

\section{Discusión}

El presente estudio analiza la percepción respecto al amor y las relaciones de pareja, así como la influencia de la socialización diferencial en la aceptación de los mitos del amor romántico en una muestra de futuros y futuras docentes. Para empezar, uno de los mitos del amor romántico más asumidos es el vinculado a la creencia "el amor todo lo puede", que fue referido tanto por hombres como por mujeres. Las personas participantes identificaron que el amor permite resolver obstáculos, por lo que fue percibido como algo positivo y una fuente de bienestar en las relaciones románticas de pareja. No obstante, de acuerdo con De Miguel (2015), los agentes de socialización ejercen una enorme influencia en las nuevas generaciones, y este tipo de imperativos podrían tener un gran impacto en el desarrollo individual de las personas y en los modos de afrontamiento ante los problemas, así como en las expectativas y formas de concebir las relaciones de pareja (Ferrer et al., 2008; Wheeler et al., 2016).

Por otra parte, la presión del entorno respecto a la forma de organización social en pareja y la idealización del amor romántico podrían estar favoreciendo la dependencia emocional (Valle y De la Villa, 2018), una percepción reportada en el presente estudio entre algunas mujeres que aludieron a no concebir una vida sin pareja o tener la necesidad de disponer de alguien que les permita desarrollar su autoestima. Esto es especialmente preocupante en una sociedad en la que se transmiten valores respecto al desarrollo de formas de conducta de dependencia en las mujeres (Bonilla y Rivas, 2018a; Ferrer et al., 2008; Ferrer y Bosch, 2013) que se sustentan en una socialización diferencial entre estas y los hombres, asignando roles distintos en múltiples contextos (Arango y Corona-Vargas, 2016; De Miguel, 2015; Vázquez y Rivas, 2017). Además, aunque algunas participantes señalaron no tener la necesidad de tener pareja para sentirse bien consigo mismas, sí refirieron que había presión por parte del entorno cuando no tenían una relación de pareja. De acuerdo con Rodríguez-Castro et al. (2015), las prescripciones sociales respecto a qué se entiende por amor podría derivar en una pérdida de la identidad propia, que se encuentra en la idealización del concepto "nosotros" en el ámbito de la pareja (Kawamichi et al., 2016; Valle y De la Villa, 2018). También se hallaron respuestas asociadas al mito "donde hay amor, hay sufrimiento", lo que implica la asunción y aceptación del malestar en la construcción de una relación afectiva, con mayor riesgo para las mujeres educadas en ser para otros (Bonilla y Rivas, 2018b). Cabe señalar la importancia que tiene la identificación de estos sesgos sociales basados en la sobrevaloración del amor teniendo en cuenta que las relaciones de pareja se inician a edades tempranas (Pazos et al., 2014) y que estas se caracterizan por ser más conflictivas que en la adultez (Shulman y Kipnis, 2001).

Por otro lado, la reciprocidad en las relaciones románticas se asocia a altos niveles de compromiso y exigencia. Esta concepción es interpretada desde los imperativos sociales, que representan niveles de exigencia diferenciados para los hombres respecto a las mujeres (Bonilla y Rivas, 2018a; Bonilla y Rivas, 2018b; Brewer y Abell, 2017; Wheeler et al., 2016). En este sentido, se han encontrado prescripciones relativas a que la reciprocidad es vital y qué sería lo mínimo que se ha de esperar en la relación, percepción reportada por un hombre. En cambio, las mujeres han proporcionado afirmaciones respecto a la manipulación emocional y el desarrollo de sentimientos de culpa cuando la correspondencia subjetiva no alcanza las cotas esperadas y no se relacionan con las expectativas de la pareja, lo que serían formas de control y de coerción (Brewer y Abell, 2015; Carton y Egan 2017; Ináncsi et al., 2015). Buena parte de la muestra no interpreta los celos como una demostración de amor, sino como una fuente de malestar tanto en las mujeres como en los varones, si bien, 
ellos manifestaron que los celos emergen siempre y que no se pueden controlar, lo que podría influir en los conflictos en las relaciones de pareja (Brewer y Abell, 2015; Latagne y Furman, 2017).

Finalmente, las mujeres participantes de la muestra señalan episodios del pasado en los que se ha ejercido contra ellas alguna forma de maltrato, de manipulación y de chantaje por parte de la pareja, que limitaba física o psicológicamente la capacidad de decisión. Cuando estas situaciones convergen con una socialización diferencial de género, no solo genera altos niveles de malestar (García-Carpintero et al., 2018), sino que es difícilmente identificable como violencia (Carton y Egan, 2017). Por todo esto, es importante la inclusión de la perspectiva de género y el desarrollo de medidas que permitan identificar la socialización diferencial, así como la incorporación de estrategias con las que desmitificar los imperativos procedentes del ideario del amor romántico en los agentes de socialización primaria. Si los y las futuras responsables de educar a las nuevas generaciones han asumido estas creencias irracionales, es necesario intervenir en la deconstrucción de tales valores haciendo que las acciones emprendidas por conseguir la igualdad sean una realidad y no una declaración de intenciones. Además, las estadísticas sobre violencia de género en España hacen que la intervención y la educación sean una prioridad (Bonilla y Rivas, 2018; Bonilla-Algovia y Rivas-Rivero, 2018; Bonilla-Algovia y Rivas-Rivero, 2019; Carbajosa y Boira, 2013).

En el presente estudio hay que identificar una serie de limitaciones. Para empezar, la muestra de participantes no guarda la misma proporción respecto al sexo. Esta circunstancia ha podido generar un mayor número de afirmaciones vinculadas al malestar en las mujeres, y que se dé un sesgo respecto al amor romántico y a tener pareja en ellas. No obstante, hay que tener en cuenta que el número de varones en los estudios de magisterio es menor, aspecto que procede en sí mismo de la socialización diferencial tradicional (Vázquez y Rivas, 2017). Por otra parte, se optó por la creación de grupos homogéneos en cuanto al sexo con la finalidad de evitar condicionamientos o que se produjese deseabilidad social entre las personas participantes. Además, esta decisión consideramos que podría facilitar el análisis respecto a la influencia de la socialización diferencial. Como futuras líneas de investigación, se podrían incorporar grupos mixtos para seguir profundizando en la influencia del entorno sobre la concepción de las relaciones de pareja y los mitos del amor romántico. Asimismo, se podrían analizar tales concepciones antes y después de incorporar formación en igualdad con el objetivo de analizar la influencia de la perspectiva de género en la construcción de la realidad social. La educación tiene un papel muy relevante en la socialización de las nuevas generaciones, por ello es necesaria la presencia en los planes de estudio de contenidos en materia de igualdad en las nuevas promociones de docentes si se quiere alcanzar la igualdad en todas las esferas de nuestra sociedad.

\section{Referencias}

Arango, M. \& Corona-Vargas, E. (2016). Guía para la igualdad de género en las políticas y prácticas de la formación docente. Organización de las Naciones Unidas para la Educación, la Ciencia y la Cultura (UNESCO). Recuperado el 6 de enero de 2020, de: https://unesdoc.unesco.org/ark:/48223/pf0000260891

Bisquert-Bover, M., Giménez-García, C., Gil-Juliá, B., Martínez-Gómez, M., \& Gil-Llario, M. D. (2019). Mitos del amor romántico y autoestima en adolescentes. International Journal of Developmental and Educational Psychology, 5(1), 507-518. http://www.infad.eu/RevistaINFAD/OJS/index.php/IJODAEP/ article/view/1633

Boisvert, S. \& Poulin, F. (2016). Romantic relationships patters from adolescence to emerging adulthood: Associations with family and peer experiences in early adolescence. Journal of Youth and Adolescence, 45(5), 945.958. https://doi.org/10.1007/s10964-016-0435-0

Bonilla, E. \& Rivas E. (2018). Propiedades psicométricas de la versión reducida de la Escala de Mitos sobre el Amor en una muestra de estudiantes colombianos. Suma Psicológica,25(2), 162-170. https://doi.org/10.14349/sumapsi.2018.v25.n2.8

Bonilla-Algovia, E. \& Rivas-Rivero, E. (2018). Mitos del amor romántico en una muestra de futuros y futuras docentes. Revista de Estudios e Investigación en Psicología y Educación, 5, 113-117. https://doi.org/10.17979/reipe.2018.5.2.3624 
Bonilla-Algovia, E., \& Rivas-Rivero, E. (2019). Creencias distorsionadas sobre la violencia contra las mujeres en docentes en formación de Colombia. Revista Colombiana de Educación, (77), 87-106. https://doi.org/10.17227/rce.num77-9571

Bosch, E., Herrezuelo, R., \& Ferrer, V. (2019). El amor romántico como renuncia y sacrificio. ¿Qué opinan los y las jóvenes? Femeris, 4(3), 184-202. https://doi.org/10.20318/femeris.2019.4935

Brewer, G. \& Abell, L. (2015). Machiavellianism in long-term relationships: competition, mate retention and sexual coercion. Scandinavian Journal of Psychology, 56, 357-362. https://doi.org/10.1111/sjop.12200

Brewer, G. \& Abell, L. (2017). Machiavellianism, relationship satisfaction, and romantic relationship quality. Europe's Journal of Psychology, 13, 491-502. https://doi.org/10.5964/ejop.v13i3.1217 Carbajosa, P. \& Boira, S. (2013). Estado actual y retos futuros de los programas para hombres condenados por violencia de género en España. Psychosocial Intervention, 22, 145-152. https://doi.org/10.5093/in2013a17

Carrasco, J. B. \& Calderero, J. F. (2007). Aprendo a investigar en educación. Madrid: RIALP.

Carretero, R., \& Nolasco, A. (2019). Sexismo y formación inicial del profesorado. Educar, 5(1), 293-310. https://dx.doi.org/10.5565/rev/educar.903

Carton, H. \& Egan, V. (2017). The dark triad and intimate partner violence. Personality and Individual Differences, 105, 84-88. https://doi.org/10.1016/j.paid.2016.09.040

De Lara, E. \& Ballesteros, B. (2007). Métodos de investigación en Educación Social. Madrid: UNED.

De Miguel, V. (2015). Percepción de la violencia de género en la adolescencia y la juventud. Informe de la Delegación del Gobierno para la Violencia de Género. Madrid: Ministerio de Sanidad, Servicios Sociales e Igualdad.

Denzin, N. \& Lincoln, Y. (2011). La investigación cualitativa como disciplina y como práctica. México: Gedisa. Fassio, A. N. (2018). Reflexiones acerca de la metodología cualitativa para el estudio de las organizaciones. Ciencias Administrativas, 6(12), 73-84. https://doi.org/10.24215/23143738e 028

Ferrer, V., Bosch, E., Navarro, C., Ramis, M. C., \& García, E. (2008). El concepto de amor en España. Psicothema, 20(4), 589-595. http://www.psicothema.com/psicothema.asp?id=3527

Ferrer, V. \& Bosch, E. (2013). Del amor romántico a la violencia de género. Para una coeducación emocional en la agenda educativa. Profesorado. Revista de currículum y formación del profesorado, 17(1), 105-122. https://recyt.fecyt.es/index.php/profesorado/article/view/41570

Forni, P. (2010). Los estudios de caso: orígenes, cuestiones de diseño y su aporte a la teoría social. Investigación en Ciencias Sociales, 3(5), 61-80.

García-Carpintero, A., Rodríguez-Santero, J., \& Porcel-Gálvez, A. (2018). Diseño y validación de la escala para la detección de violencia en el noviazgo en jóvenes de la Universidad de Sevilla. Gaceta Sanitaria, 32, 109-202. https://doi.org/10.1016/j.gaceta.2017.09.006

Gil, J. A. (2011). Técnicas e instrumentos para la recogida de información. Madrid: UNED.

González, E. \& Molina, T. (2018). Relaciones románticas tempranas y su asociación con comportamientos de riesgo en la salud durante la adolescencia. Revista Chilena de Obstetricia y ginecología, 85(4), 368-376. https://doi.org/10.4067/s0717-752620180004000368

Ináncsi, T., Lang, A., \& Bereczkei, T. (2015). Machiavellianism and Adult Attachment in General Interpersonal relationships and Close relationships. Europe's Journal of Psychology, 11, 139-154. https://doi.org/10.5964/ejop.v11i1.801

Kawamichi, H., Sugawara, S. K., Hamano, Y., Makita, K., Matsunaga, M., Tanabe, H., ... Sadato, N. (2016). Being in a romantic relationship in associated with reduced gray matter density in striatum and increased subjective happiness. Frontiers in Psychology, 7, 1763. https://doi.org/10.3389/fpsy.g.2016.01763

Wheeler, L., Killoren, S., Whiteman, S., Updegraff, K., \& McHale, S. (2016). Romantic Relationship Experiences from Late Adolescence to Young adulthood: The role of sibling in Mexican-Origin Families. Journal of Youth and Adolescence, 45, 900-915. https://doi.org/10.1007/s10964-015-0392-z

Lantagne, A. \& Furman, W. (2017). Romantic relationship development: The interplay between age and relationship length. Developmental Psychology, 53, 1738-1749. https://doi.org/10.1037/dev0000363 
Papp, L. J., Liss, M., Erchull, M. J., Godfrey, H., \& Waaland-Kreutzer, L. (2017). The dark side of heterosexual romance: endorsement of romantic beliefs relates to intimate partner violence. Sex Roles, 76(1-2), 99-109. https://doi.org/10.1007/s1199-016-0668-0

Pascual, A. (2016). Sobre el mito del amor romántico. Amores cinematográficos y educación. Dedica. Revista Educação e Humanidades, 10, 63-78. https://revistaseug.ugr.es/index.php/dedica/article/view/6850

Patton, M. (1987). How to Use Qualitative Methods in Evaluation. California: SAGE.

Pazos, M., Oliva, A., \& Hernando, A. (2014). Violencia en relaciones de pareja de jóvenes y adolescentes. Revista Latinoamericana de Psicología, 46(3), 148-159. https://doi.org/10.1016/S0120-0534(14)70018-4

Rivas, E. \& Bonilla, E. (2020). Salud mental y miedo a la separación en víctimas de violencia de pareja. Revista Iberoamericana de Psicología y Salud, 11(1), 54-67. https://doi.org/10.23923/j.rips.2020.01.035

Rivas, E., Bonilla, E., \& Vázquez, J. J. (2020). Risk factors associated with substance use in female victims of abuse living in a context of poverty. Anales de Psicología, 36(1), 173-180. https://doi.org/10.6018/analesps.362541

Rodríguez-Castro, Y., Lameiras-Fernández, M., \& Carrera, V. (2015). Amor y sexismo: una peligrosa relación en los y las adolescentes gallegos/as. Revista de Estudios e Investigación en Psicología y Educación, Extr.(2), 2-14. https://doi.org/10.17979/reipe.2015.0.02.234

Subirats, M. (2017). Coeducación, apuesta por la libertad. Barcelona: Octaedro.

Shulman, S. \& Kipnis, O. (2001). Adolescent romantic relationships: A look from the future. Journal of Adolescence, 24, 337-351. https://doi.org/10.1006/jado.2001.0409

Valle, L. \& De la Villa, M. (2018). Dependencia emocional y estilo de apego adulto en las relaciones de noviazgo en jóvenes españoles. Revista Iberoamericana de Psicología y Salud, 9(1), 27-41. https://doi.org/10.23923/j.rips.2018.01.013

Vázquez, J. J. \& Rivas, E. (2017). Estereotipos de género. En E. Carmona \& I. Garrido (Coord.), Diversidad de Género e Igualdad de Derechos (pp. 19-31). Valencia: Tirant lo Blanch.

Fecha de recepción: 12 de octubre de 2019.

Fecha de revisión: 10 de abril de 2020.

Fecha de aceptación: 13 de abril de 2020.

Fecha de publicación: 1 de julio de 2020. 